\title{
Negative bias stress of MOS devices at high electric fields and degradation of MNOS devices
}

Kjell O. Jeppson and Christer M. Svensson

Citation: Journal of Applied Physics 48, 2004 (1977); doi: 10.1063/1.323909

View online: http://dx.doi.org/10.1063/1.323909

View Table of Contents: http://aip.scitation.org/toc/jap/48/5

Published by the American Institute of Physics

\section{Articles you may be interested in}

Negative bias temperature instability: Road to cross in deep submicron silicon semiconductor manufacturing Journal of Applied Physics 94, 1 (2003); 10.1063/1.1567461

Dynamic recovery of negative bias temperature instability in p-type metal-oxide-semiconductor field-effect transistors

Applied Physics Letters 83, 1647 (2003); 10.1063/1.1604480

Simple technique for separating the effects of interface traps and trapped-oxide charge in metal-oxidesemiconductor transistors

Applied Physics Letters 48, 133 (1998); 10.1063/1.96974

Effects of oxide traps, interface traps, and "border traps" on metal-oxide-semiconductor devices Journal of Applied Physics 73, 5058 (1998); 10.1063/1.353777

Bias-stress-induced stretched-exponential time dependence of charge injection and trapping in amorphous thinfilm transistors

Applied Physics Letters 62, 1286 (1998); 10.1063/1.108709

Mechanism of negative-bias-temperature instability

Journal of Applied Physics 69, 1712 (1998); 10.1063/1.347217

\section{AID Jomanalor \\ AlP | Applied Physics}

Save your money for your research.

It's now FREE to publish with us -

no page, color or publication charges apply.
Publish your research in the

Joumal of Applied Physics

to claim your place in applied

physics history. 


\title{
Negative bias stress of MOS devices at high electric fields and degradation of MNOS devices
}

\author{
Kjell O. Jeppson and Christer M. Svensson \\ Research Laboratory of Electronics, Chalmers University of Technology, Fack, S-402 20 Göteborg, Sweden \\ (Received 16 August 1976; accepted for publication 13 December 1976) \\ One of the most important degradation effects observed in MNOS memory transistors is a negative shift \\ of the threshold window. This negative shift is caused by a strong increase of the density of $\mathrm{Si}_{-} \mathrm{SiO}_{2}$ surface \\ traps. This effect has been proposed to be caused by the same effect that is observed in MOS devices \\ subjected to negative-bias stress (NBS). In this paper, a detailed study of the increase of the number of \\ surface traps in MOS structures after NBS at temperatures $\left(25-125^{\circ} \mathrm{C}\right)$ and fields $(400-700 \mathrm{MV} / \mathrm{m})$ \\ comparable to those used in MNOS devices is presented. Two different behaviors are observed. At low \\ fields the surface-trap density increases as $t^{1 / 4}$ and at high fields it increases linearly with the stress time $t$. \\ The low-field behavior is temperature and field dependent and the zero-field activation energy is \\ determined to be $0.3 \mathrm{eV}$. The high-field behavior is strongly field dependent but independent of \\ temperature. A physical model is proposed to explain the surface-trap growth as being diffusion controlled \\ at low fields and tunneling limited at high fields. A comparison with MNOS degradation is made and it \\ was found to be related to the $t^{1 / 4}$ behavior mentioned above.
}

PACS numbers: 73.40.Qv, 73.20. $-\mathrm{r}, 72.20 . \mathrm{Ht}$, 85.30.De

\section{INTRODUCTION}

The use of the MNOS nonvolatile memory device as a read/write memory is limited to a certain number of write/erase cycles $\left(\sim 10^{6}-10^{12}\right.$ cycles $) .^{1,2}$ Frequent write/erase cycles introduce changes in device properties due to the high oxide field $(700-1600 \mathrm{MV} / \mathrm{m})$. The two most important degradation effects observed in $p$ channel MNOS transistors are a negative shift of the threshold window (up to $10 \mathrm{~V}$ ) and a decrease in the retention time. The negative shift of the threshold window is caused by a large increase in the density of the $\mathrm{Si}-\mathrm{SiO}_{2}$ surface traps. ${ }^{1-3}$

It is well known that surface traps are created at the $\mathrm{Si}-\mathrm{SiO}_{2}$ interface of an MOS capacitor when negative bias is applied to the gate at elevated temperatures. ${ }^{4,5}$ This negative-bias stress (NBS) effect has been proposed to be the same effect that causes the degradation of MNOS devices repeatedly operated at high write/erase gate voltages. ${ }^{1}$

The NBS effect at elevated temperature $\left(250-300^{\circ} \mathrm{C}\right)$ was examined by Deal et al. ${ }^{4}$ and by Goetzberger et al. ${ }^{5}$ They found that both the surface-trap density $N_{\text {st }}$ and the surface-charge density $Q_{o x}$ increased upon negativebias stress. The increases of $N_{s t}$ and $Q_{0 x}$ were very similar and were found to be logarithmic in time. Furthermore, the increase was nearly proportional to the applied field $(0-300 \mathrm{MV} / \mathrm{m})$ and was larger at higher temperatures.

This work extends the measurements on the NBS effect in MOS capacitors to temperatures $\left(25-125^{\circ} \mathrm{C}\right)$ and fields $(400-700 \mathrm{MV} / \mathrm{m})$ more typical for the operation of MNOS devices. When an MOS capacitor is subjected to NBS it is not too surprising that charge may be trapped at the $\mathrm{Si}-\mathrm{SiO}_{2}$ interface. It is much more interesting that new surface traps are actually created during NBS. We believe that this may not happen unless the atomic structure is changed at the interface. Therefore, our measurements are focused on the surface traps. The NBS behavior of MOS capacitors is characterized through studies of the time dependence of the surface-trap formation at different stress voltages and temperatures. Two mechanisms are observed to create new surface traps during NBS. The first mechanism, which is believed to be diffusion controlled, is presented schematically and the time dependence of this type of process is shown to agree with experiments. The other mechanism, which is dominant at very high fields, is a hole tunneling process. After evaluation of the measurements, possible electrochemical reactions which could occur at the $\mathrm{Si}_{-} \mathrm{SiO}_{2}$ interface are discussed. Finally, the NBS behavior of MOS capacitors is compared to the degradation behavior of MNOS capacitors which is more complex to evaluate.

\section{SAMPLE PREPARATION}

The NBS measurements were performed on MOS samples fabricated on $\langle 100\rangle 2-3-\Omega \mathrm{cm} n$-type silicon wafers. A typical high-quality MOS sample was first cleaned in $\mathrm{H}_{2} \mathrm{O}_{2}-\mathrm{NH}_{3}$ and $\mathrm{H}_{2} \mathrm{O}_{2}-\mathrm{HCl}$, and then rinsed in double-distilled water water followed by a $30-\mathrm{s}$ dip in $48 \% \mathrm{HF}$ and a final short rinse in double-distilled water. An oxide layer of about $950 \AA$ was grown in dry oxygen in a resistance-heated furnace $\left(1200^{\circ} \mathrm{C}, 12\right.$ min). The metal gate contacts were e-gun-evaporated aluminum contacts in order to reduce the $\mathrm{Na}^{+}$ion content. The electrodes are circular dots with a $700-\mu \mathrm{m}$ diameter (area $3.85 \times 10^{-7} \mathrm{~m}^{2}$ ). Radiation damage was annealed in forming gas $\left(10 \% \mathrm{H}_{2}, 90 \%, \mathrm{~N}_{2}\right)$ for $10 \mathrm{~min}$ at $500^{\circ} \mathrm{C}$

For comparison of the NBS effect in MOS samples with the degradation effect in MNOS memory devices, MNOS capacitors were fabricated and exposed to highfield stress. The fabrication process of these MNOS samples has been described elsewhere. ${ }^{6}$

The MNOS samples were fabricated on $\langle 100\rangle 0.8-1.2-$ $\Omega \mathrm{cm} n$-type silicon wafers. These were cleaned the same way as above but dipped for $2 \mathrm{~min}$ in $48 \% \mathrm{HF}$ before a $30-s$ rinse in double-distilled water. At this stage the thickness of the residual oxide was $8-10 \AA$ according to ellipsometer measurements. A thin oxide 


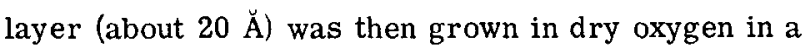
resistance-heated furnace at a temperature of about $600^{\circ} \mathrm{C}$ for $15 \mathrm{~min}$. The oxide thickness was again measured by ellipsometer, after which the slice was placed in the nitride reactor. The nitride layer was deposited at $725^{\circ} \mathrm{C}$ and was then annealed in argon at $900^{\circ} \mathrm{C}$ for $15 \mathrm{~min}$. The metal gates on the MNOS devices were $250 \times 500-\mu \mathrm{m}\left(1.25 \times 10^{-7} \mathrm{~m}^{2}\right) \mathrm{Cr}-\mathrm{Au}$ dots.

\section{MEASUREMENTS AND EVALUATION OF NEGATIVE-BIAS STRESS}

Negative-bias stress (NBS) of an MOS capacitor generally means that a negative voltage is applied to the metal gate for a certain time at a certain stress temperature in the range from room temperature ( $\mathrm{RT}$ $=25^{\circ} \mathrm{C}$ ) up to $300^{\circ} \mathrm{C}$. In our experiments the temperature was limited to the range $25-125^{\circ} \mathrm{C}$ which is typical for the operation of Mas devices. The voltage range was $40-66 \mathrm{~V}$ corresponding to $400-700 \mathrm{MV} / \mathrm{m}$ on our $950-\AA$ samples. After stress, the sample is cooled to room temperature with the gate floating. Changes in the interface properties are measured with the $C-V$ technique, which includes recording of a high-frequency and a quasistatic low-frequency capacitance curve.

A standard experimental setup for stress and $C-V$ measurements was used. The MOS capacitor was placed on a nickel pedestal which was resistively heated to the stress temperature. Good electrical and thermal contact to the back side was provided by a thin layer of In-Ga which is liquid at RT. The stress voltage was applied to the metal gate through a thin gold wire probe. After stress the sample was cooled to RT and the highfrequency (1 $\mathrm{MHz}$ ) capacitance was measured with a Boonton 72B Capacitance Bridge. The low-frequency quasistatic $C-V$ curve was measured with a Keithley $610 \mathrm{C}$ Electrometer using the slow-ramp technique (sweep rate $\sim 60 \mathrm{mV} / \mathrm{s}$ ), 7,8

From the measured $C-V$ plots, the surface-trap density $N_{\mathrm{st}}$ may be calculated from the following relationship?

$$
N_{\mathrm{st}}=\frac{C_{\mathrm{ox}}}{q A} N_{r},
$$

where

$$
N_{r}=\frac{C_{\mathrm{LF}} / C_{\mathrm{ox}}}{1-C_{\mathrm{LF}} / C_{\mathrm{ox}}}-\frac{C_{\mathrm{HF}} / C_{\mathrm{ox}}}{1-C_{\mathrm{HF}} / C_{\mathrm{ox}}},
$$

$C_{o x}$ is the oxide capacitance, $q$ is the electronic charge, $A$ is the MOS-capacitor area, $N_{r}$ is the relative surface-trap density, $C_{L F}$ is the quasistatic capacitance, and $C_{\mathrm{HF}}$ is the $1-\mathrm{MHz}$ capacitance.

If the $\mathrm{C}-\mathrm{V}$ plots are recorded on a normalized scale, the relative surface-trap density $N_{r}$ is easily calculated and the values of $N_{r}$ are sufficient in order to study the changes of the surface-trap density. The smallest measureable value of $N_{r}$ is about 0.04 . With an oxide thickness of $950 \AA, C_{\text {ox }} / q A=2.2 \times 10^{11} \mathrm{~cm}^{-2} \mathrm{eV}^{-1}$, which means that surface-trap densities $>10^{10} \mathrm{~cm}^{-2} \mathrm{eV}^{-1}$ may be determined with this method.

A typical set of $\mathrm{C}-\mathrm{V}$ curves recorded with increasing accumulated stress time as parameter is shown in $\mathrm{Fig}$.
1. Normally, only the relative surface-trap density at midband was evaluated from such curves. The changes in the midband relative surface-trap density during negative-bias stress is discussed in more detail in Secs. IV-IX.

Selected measurements were fully evaluated using a Wang 600 to calculate the energy distribution of the surface-state density. The energy range is limited on one side by the flatband condition, and on the other side by the onset of inversion. Near flatband, the highfrequency capacitance curve is influenced by the surface traps since their time constants become too short. The surface potential was calculated through integration of the low-frequency curve as described by Berglund. ${ }^{9}$ The undetermined additive constant was determined through calculation of the ideal midband capacitance. This determination agreed very well with the one obtained when the integrated energy range was adjusted symmetrically to the middle of the energy band. The inversion and flatband points were easily determined since they lie $\pm 0.3 \mathrm{eV}$ from midband (for a doping concentration of $1.5 \times 10^{15} \mathrm{~cm}^{-3}$ ).

The surface potential is most accurately determined from the $\mathrm{C}-\mathrm{V}$ plots measured before stress. Since the relationship between the surface potential and the highfrequency capacitance is independent of the number of surface traps, it is not necessary to integrate the lowfrequency curve after each stress cycle. A full evaluation of the stress results shown in Fig. 1, following these guidelines, is presented in Fig. 2. The increase in the surface-trap density as a result of negative-bias stress is shown in the energy range from flatband to inversion.

\section{BEHAVIOR OF SURFACE TRAPS}

The time dependence of the surface-trap generation during negative-bias stress was studied at different stress voltages and temperatures. As mentioned above, our attention was focused on the relative surface-trap

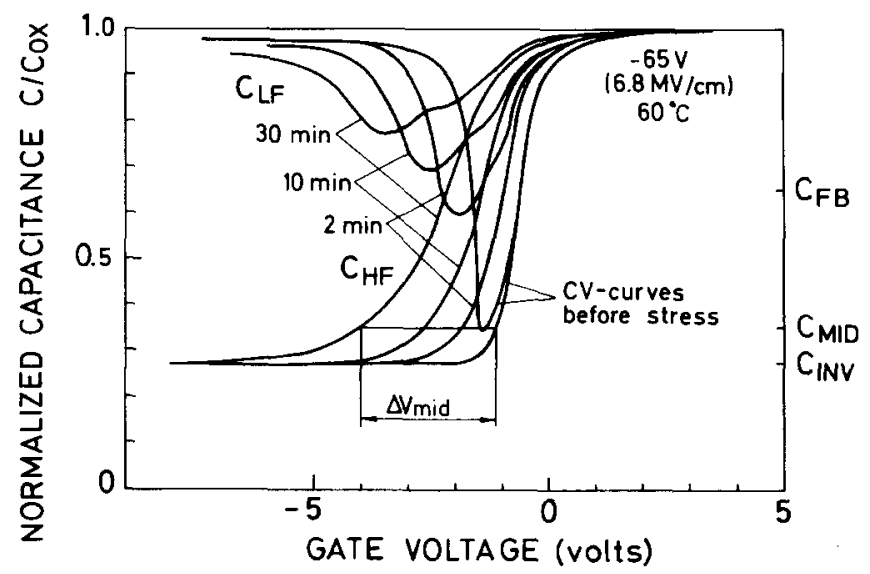

FIG. 1. A typical set of $C-V$ curves recorded with increasing accumulated stress time as parameter. The right-most curve pair is recorded before stress and indicates low surface-trap density. As stress time increased (curves are recorded after 2,10 , and $30 \mathrm{~min}$ of NBS) the quasistatic $\left(C_{\mathrm{LF}}\right)$ and the $1-\mathrm{MHz}$ $\left(C_{\mathrm{HF}}\right) C-V$ curves diverge and indicate an increasing surfacetrap density. 


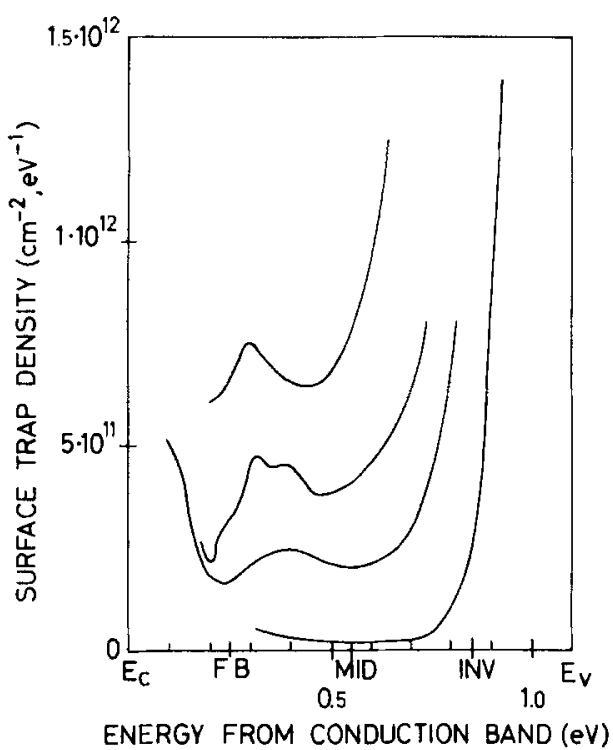

FIG. 2. A full evaluation of the stress results in Fig. 1 showing the energy distribution of the surface-trap density and its increase after NBS with stress time as parameter.

density at midband, which made data evaluation fast and simple.

The most extensive stress measurements were performed on the same high-quality MOS sample. For this sample the stress field was in the range $4-7 \mathrm{MV} / \mathrm{cm}$, where the upper limit was set by destructive breakdown of the MOS structure. Measurements were made at three different stress temperatures 25,60 , and $125^{\circ} \mathrm{C}$. Some results are shown in Fig. 3. Each curve was obtained from a separate capacitor on the same sample (oxide thickness $950 \AA$ ).

When the NBS effect was examined by Deal et al., they studied the increase of the fixed oxide charge. They found that this increase generally was accompanied by an equal amount of growth in the number of surface traps. As mentioned above, our measurements are focused on the number of surface traps, but they confirm the observation ${ }^{4,5}$ that there is an equal growth

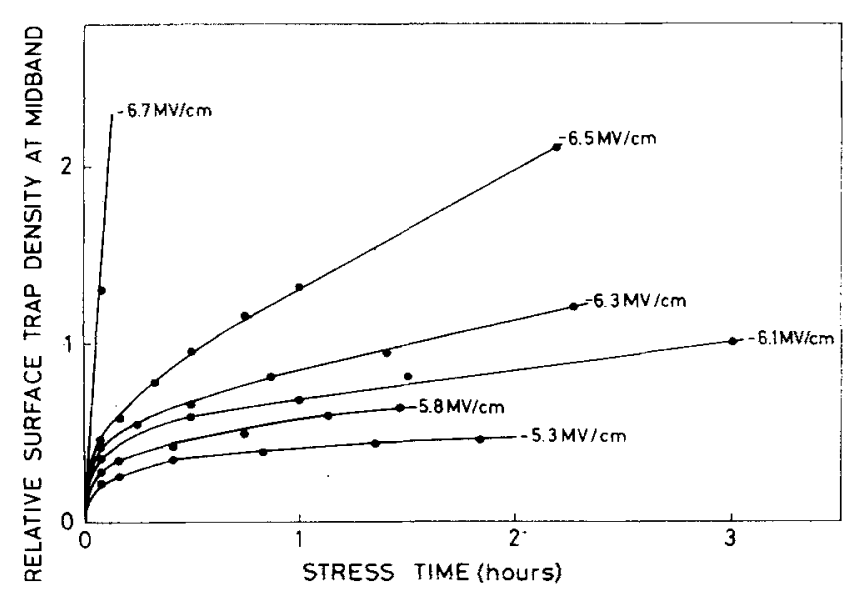

FIG. 3. Relative surface-trap density at midband and its increase with stress time for different NBS fields at room temperature $\left(25^{\circ} \mathrm{C}\right)\left(d_{\mathrm{ox}}=950 \AA\right)$.

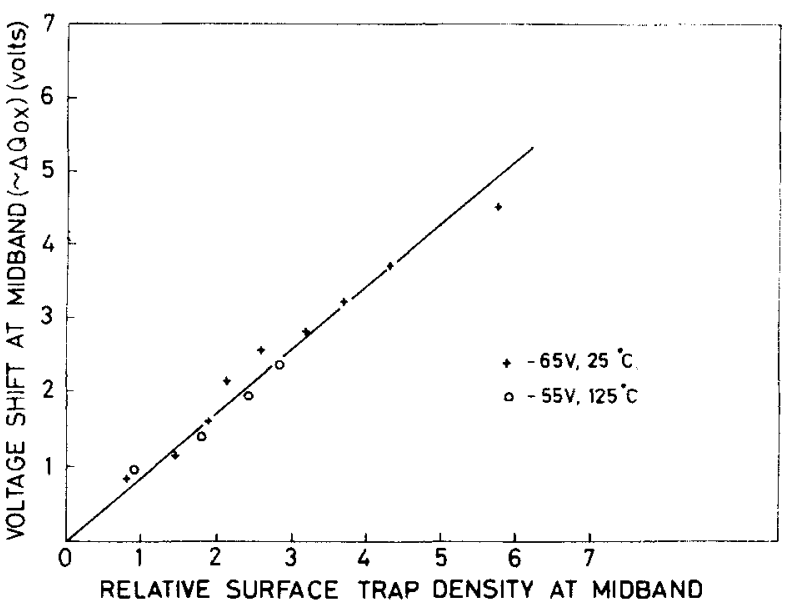

FIG. 4. The increase of the oxide charge measured as the shift of the $C-V$ curve at midband, plotted against the relative surface-trap density at midband. The same relationship is observed after NBS at both $125^{\circ} \mathrm{C},-55 \mathrm{~V}(5.8 \mathrm{MV} / \mathrm{cm})$ and $25^{\circ} \mathrm{C},-65 \mathrm{~V}(6.8 \mathrm{MV} / \mathrm{cm})$ and indicates that there is roughly one surface trap per oxide charge.

of the fixed oxide charge and the surface-trap density independent of the NBS conditions. A typical plot of the growth of the fixed oxide charge, measured as the midband voltage shift, versus the midband surface-trap density is shown in Fig. 4. This plot shows the same relationship independent of NBS fields and temperatures.

The surface traps that are created during NBS can be removed by annealing. If a capacitor which has been exposed to NBS at a certain stress temperature remains at that temperature with the gate grounded, the number of surface traps slowly decreases to a new level of surface-trap density. If the annealing temperature is then increased, the number of surface traps further decreases to a new level as depicted in Fig. 5 .

After NBS, even with very short stress times, some capacitors showed a strongly increased leakage current during recording of the quasistatic $\mathrm{C}-\mathrm{V}$ curve. This phenomenon occurred following stress at very high voltage, particularly at room temperature. However, if such a capacitor was left with the gate voltage sweep-

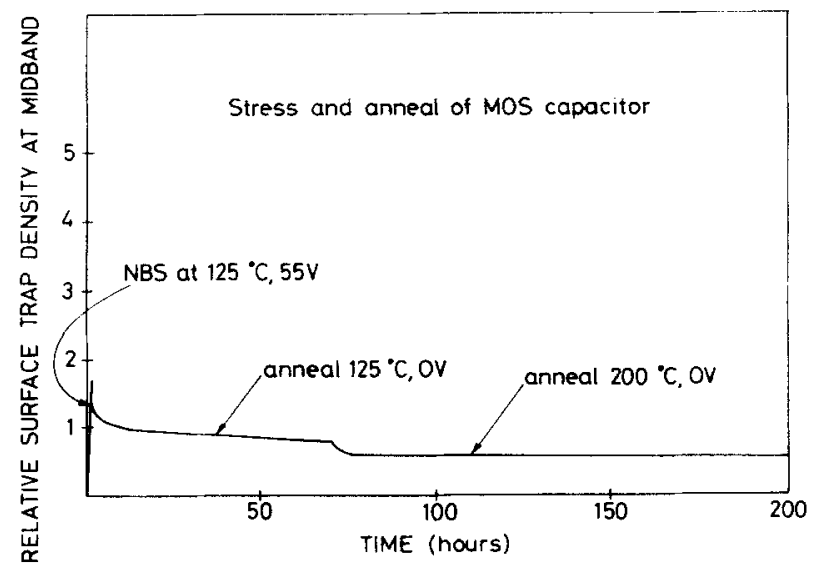

FIG. 5. The behavior of an MOS capacitor that is stressed at $125^{\circ} \mathrm{C},-55 \mathrm{~V}$ for a short time and thereafter annealed at 125 and $200^{\circ} \mathrm{C}$. The relative surface-trap density is shown against time. 


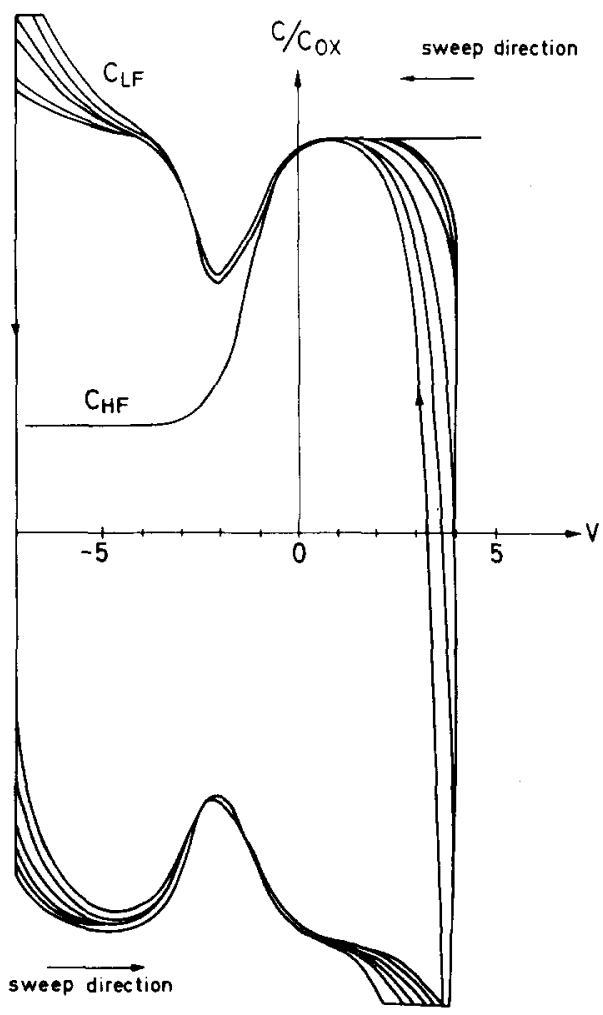

FIG. 6. Quasistatic $C-V$ curves recorded after $10 \mathrm{~min}$ of NBS with $-65 \mathrm{~V}$ at $25^{\circ} \mathrm{C}$. These recordings are a few of the set of curves that were recorded continuously during $1 \mathrm{~h}$ after stress. They show a leakage current that is decreasing with time. Is this a hydrogen-ion leakage current?

ing back and forth while the quasistatic $\mathrm{C}-\mathrm{V}$ curve was continuously recorded, the leakage current slowly disappeared, as shown in Fig. 6. This leakage current may be an ion drift current, possibly by hydrogen ions.

\section{DISCUSSION OF MEASUREMENTS}

To our knowledge, no model for surface-trap generation has been published. Surface traps are usually believed to be either induced by charge trapped at the interface, or due to broken chemical bonds. Since the charge model is very vague in explaining surface traps in the middle of the bandgap, we believe that surface traps cannot be formed unless the atomic structure is changed at the interface. Therefore we propose a chemical model for formation of surface traps.

Goetzberger et al. ${ }^{5}$ found that the surface-trap density increased as the logarithm of time at 250 and $300^{\circ} \mathrm{C}$. Their reason for choosing a logarithmic plot is according to Walden's ${ }^{10}$ theory of charge trapping in an insulator. However, Walden is mathematically treating electron (or hole) injection to traps located well inside the insulator and this phenomena is not directly connected with the generation of surface traps.

Based on our measurements, we believe this generation to be diffusion controlled, as discussed later. Our mathematical treatment gives a $t^{1 / 4}$ time dependence. This is very similar to a logarithmic behavior over a few decades, so the data of Goetzberger et al. also fit this time dependence.
Our measured values are plotted against $\ell^{1 / 4}$ in $\mathrm{Fig}$. 7 for 25 and $125^{\circ} \mathrm{C}$ with the stress field as parameter. It is clearly seen that $N_{\mathrm{st}}$ increases as $t^{1 / 4}$ at $125^{\circ} \mathrm{C}$ which is in agreement with Goetzberger's high-temperature results. At room temperature the $t^{1 / 4}$ dependence is valid for low stress voltages. For high stress voltages it is valid only during a limited initial stress time. For stress fields larger than $6.3 \mathrm{MV} / \mathrm{cm}$ the $t^{1 / 4}$ dependence is no longer valid. Instead the formation of surface traps becomes linear in time.

\section{MODEL FOR NBS EFFECT}

In our model we assume that the silicon interface contains a large number of defects which are electrically inactive, but may become electrically active upon stress. This activation is assumed to take place through a chemical reaction. The existence of similar electrochemical reactions has been proved by Nicollian et al. ${ }^{11}$

A scheme which explains the $t^{1 / 4}$ mechanism is (surface defect) $\rightleftharpoons($ surface trap $)+(\text { surface charge })^{+}$

$$
+X_{\text {interface }}+e^{-} \text {(to the silicon) }
$$

$X_{\text {interf ace }} \stackrel{\text { diffusion }}{\rightleftharpoons} X_{\text {bulk }}$.

In this process a diffusing species $X$ is formed at the interface when the surface defect is electrically activated. A surface trap and a surface charge are then left at the defect site in fixed positions close to each other. The process is field dependent since a transfer of charge takes place. It agrees with the observations that equal numbers of surface traps and surface charges are produced.

The observed $t^{1 / 4}$ behavior of the surface-trap density $N_{\text {st }}$ suggests that the survival of a created surface trap is diffusion controlled. This means that a stable surface trap is only formed if the species $X$ diffuses away from the interface into the oxide (see the Appendix).

If the process is controlled by the rate at which $X$ diffuses away from the interface into the oxide, it may be shown (this is done in the Appendix) that the surfacetrap density increases as

$$
N_{\mathrm{st}}=R_{1} t^{1 / 4}
$$

Here, $t$ is the stress time and $R_{1}$ is the rate constant of surface-trap formation. For the relative surfacetrap density $N_{r}$ we may write

$$
N_{r}=R_{1}^{\prime} t^{1 / 4}
$$

where $R_{1}^{\prime}=\left(q A / C_{\text {ox }}\right) R_{1}$ is the relative rate constant.

The time dependence of this process thus explains our observations at low fields. However, in this schematical process the actual defects have not yet been identified. Since infrared measurements have shown that large numbers of $\mathrm{Si}-\mathrm{H}$ groups exist in the bulk $\mathrm{SiO}_{2}$ and probably also in very large numbers at the interface, ${ }^{12,13}$ we believe that the surface defect in scheme (3) is an $\equiv \mathrm{Si}_{s}-\mathrm{H}$ group at the silicon interface. ( $\mathrm{Si}_{s}$ indicates a silicon surface atom bonded to three other silicon atoms.) For the activation of this defect we 

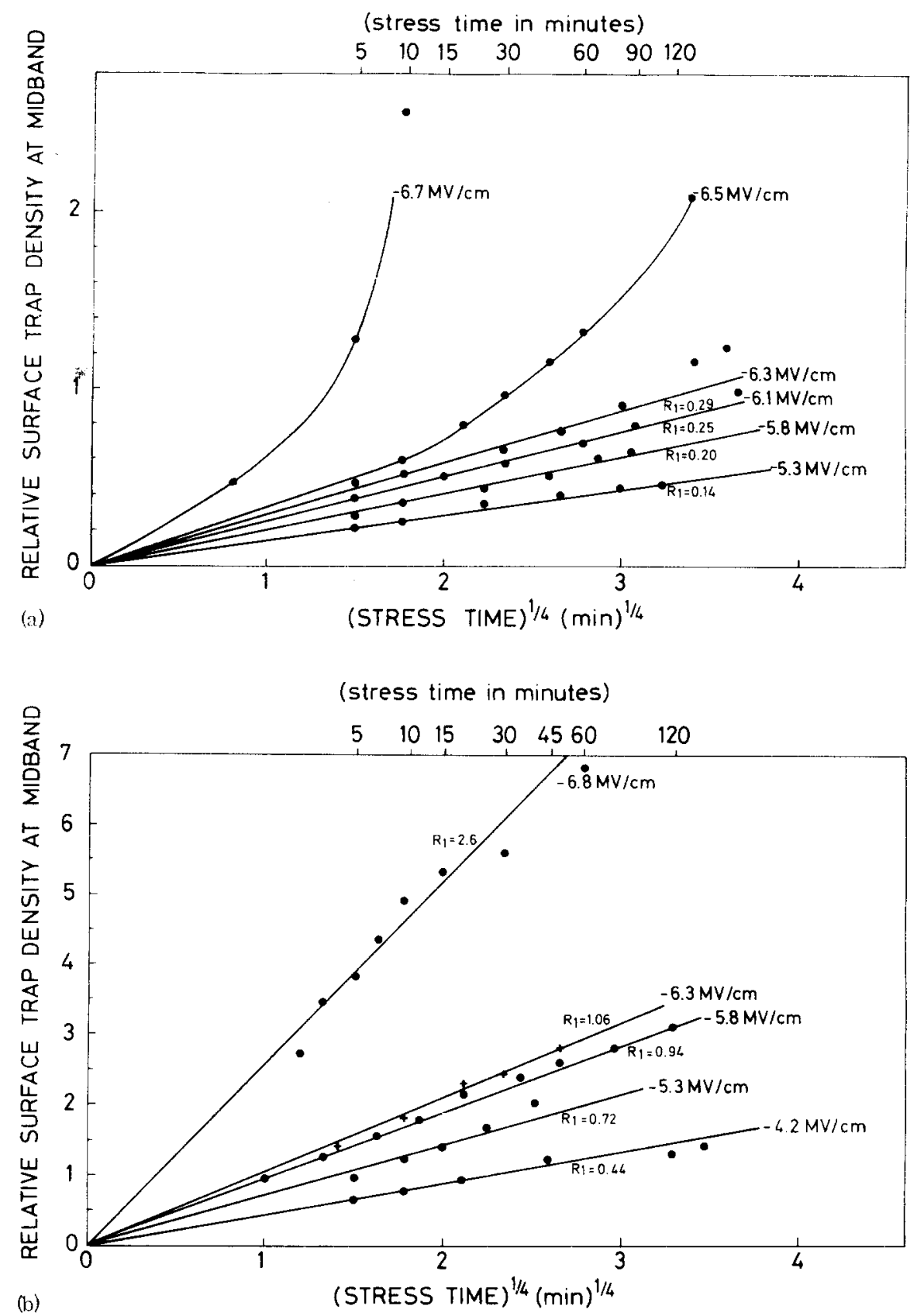

FIG. 7. Relative surface-trap density at midband plotted against (stress time) ${ }^{1 / 4}$ for different NBS fields $\left(d_{\mathrm{cx}}=950 \AA\right)$. (a) at room-temperature $\left(25^{\circ} \mathrm{C}\right),(\mathrm{b})$ at $125^{\circ} \mathrm{C}$.

propose the following reaction which is based on a discussion by Revesz ${ }^{14}$ on possible chemical reactions at the interface of a $\mathrm{Si}-\mathrm{SiO}_{2}$ system. This reaction is equivalent to process (3):

$$
\begin{aligned}
& \equiv \mathrm{Si}_{s}-\mathrm{H}+\equiv \mathrm{Si}_{o}-\mathrm{O}-\mathrm{Si}_{o} \equiv \rightleftharpoons \equiv \mathrm{Si}_{s}^{+}+\equiv \mathrm{Si}_{o}^{+}+\equiv \mathrm{Si}-\mathrm{OH}+e^{-},
\end{aligned}
$$

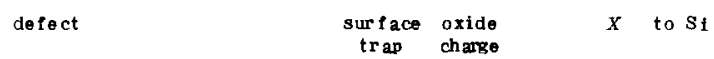

where $\mathrm{Si}_{o}$ is a silicon atom in the oxide.

When the defect is activated, the hydrogen, which is weakly bonded to the $\mathrm{Si}_{s}$ atom, reacts with the $\mathrm{SiO}_{2}$ and forms an $\mathrm{OH}$ group bonded to an oxide $\mathrm{Si}_{o}$ atom, leaving one trivalent $\mathrm{Si}_{a}^{+}$in the oxide and one trivalent $\mathrm{Si}_{s}^{*}$ at the silicon surface. This chemical reaction is schematically represented in a two-dimensional model in Fig. 8.
The credibility of this reaction is supported by many facts. The silicon surface trivalent $\mathrm{Si}_{s}^{*}$ is assumed to form the surface trap, in agreement with many earlier suggestions. ${ }^{14-16}$ The $\mathrm{Si}_{o}^{+}$is a charged trivalent oxide Si atom bonded to three oxygens. Such a defect is a good candidate for the oxide charge defect. ${ }^{14,17}$ The silanol group Si-OH finally is known to be a relatively fast-diffusing group in $\mathrm{SiO}_{2}$ and is normally introduced into the oxide by water decomposition. ${ }^{11-13}$ Further more, the diffusion constant is known for $\mathrm{OH}$ in $\mathrm{SiO}_{2}$; using the data given in Ref. $13, D \approx 2.5 \times 10^{-18} \mathrm{~m}^{2} / \mathrm{s}$ at $125^{\circ} \mathrm{C}$. This may be compared to the limit of validity of our theory, $D \leqslant W^{2} / 4 t=10^{-18} \mathrm{~m}^{2} / \mathrm{s}$ (see the Appendix). It is clear that $\mathrm{OH}$ is a good candidate for our $X$. We may also note that the diffusion constant of hydrogen in $\mathrm{SiO}_{2}$ is expected to be considerably larger. A value of $10^{-13} \mathrm{~m}^{2} / \mathrm{s}$ at $125^{\circ} \mathrm{C}$ can be deduced from data in Ref. 18 (probably $\mathrm{H}_{2}$ diffusion) and a large value of the dif- 


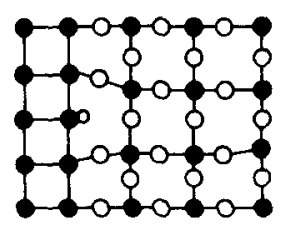

$\equiv \mathrm{Si}_{\mathrm{S}}-\mathrm{H}$ defect

(a)

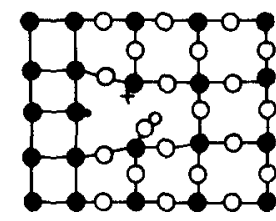

(b)

$\equiv \mathrm{Si}_{\mathrm{S}}+=\mathrm{Si}_{\mathrm{O}^{+}}^{+} \equiv \mathrm{Si}-\mathrm{OH}$

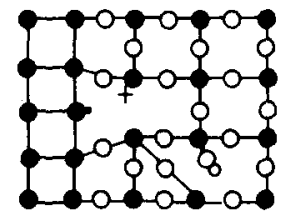

(c)

diffusing $\mathrm{OH}$-group

- Silicon

O Oxygen

- Hydrogen

FIG. 8. Schematic two-dimensional representation of the $\mathrm{Si}-\mathrm{SiO}_{2}$ interface, showing (a) the $\equiv \mathrm{Si}-\mathrm{H}$ defect, and (b) how this defect may be electrically activated during NBS to form a surface trap, an oxide charge, and a hydroxyl group, that (c) may diffuse in the oxide and be the reaction-limiting factor. $\left(\mathrm{Si}_{s}\right.$ means a silicon atom at the surface bonded to other silicon atoms, while $\mathrm{Si}_{0}$ means a silicon atom in the oxide.)

fusion constant of $\mathrm{H}$ is necessary to explain why the electrochemical charging effect observed by Nicollian et al. ${ }^{11}$ was not diffusion limited.

According to reaction (5), the surface charge is located in the oxide a distance $a$ from the silicon surface. Therefore we expect the enthalpy of the reaction to decrease in an electric field $E_{s}$, since an electron is transferred from the oxide-charge position into the silicon. The energy $q a E_{s}$ gained by the electron is reflected in the expression for the rate constant (see the Appendix)

$$
R_{1}^{\prime}=R_{10}^{\prime} \exp \left[-\left(q / k T\left(\phi_{0}-\frac{1}{2} a E_{s}\right)\right]\right.
$$

where $\phi_{0}$ is the zero-field activation energy and $R_{10}^{\prime}$ is a constant. For the NBS conditions in Fig. 7 where the $t^{1 / 4}$ behavior is valid, the slope of the straight line was taken as the rate constant of surface-trap formation. This rate, $R_{1}^{\prime}$, is plotted in Fig. 9 as a function of stress field with the stress temperature as parameter.

The field and temperature dependence found in Fig. 9 agrees very well with the diffusion theory [Eq. (6)]. From Fig. 9 we obtain $a=3.2 \AA$, and by replotting the data against $1 / T$ we estimate $\phi_{0}=0.3 \mathrm{eV}$. These values are compatible with the above model since $a$ is expected to be about $3.1 \AA$, which is the $\mathrm{Si}-\mathrm{O}-\mathrm{Si}$ distance in $\mathrm{SiO}_{2}$ ( $\phi_{0}$ is discussed in the Appendix).

\section{ANNEALING OF CREATED SURFACE TRAPS}

According to the above model, the created surface traps can only be annealed out again if the species $X$ diffuses back to the interface. Therefore, the annealing process will also be diffusion limited. According to the Appendix, we expect the surface-trap density after degradation to decrease as

$$
N_{\mathrm{st}}=R_{1}\left[t^{1 / 4}-\left(t-t_{d}\right)^{1 / 4}\right], \quad l=t_{d},
$$

where degradation is assumed to start at $t=0$ and stop at $t=t_{d}$.

However, this formula assumes that each surface trap is formed when a species $X$ diffuses away from the interface and that each of these can return to their original site. Probably it is more reasonable to assume that only a fraction $\gamma$ of the formed surface traps can be annealed by this mechanism. The remaining fraction $1-\gamma$ either corresponds to species $X$ which have been trapped in the oxide (e.g., by chemical bonding to other oxide defects) or to surface traps that were created by other mechanisms (this will be discussed in Secs. VIII and IX). We would therefore expect $N_{\mathrm{st}}$ to anneal out as

$$
N_{\mathrm{st}}=\gamma R_{1}\left[t^{1 / 4}-\left(t-t_{d}\right)^{1 / 4}\right]+(1-\gamma) R_{1} t_{d}^{1 / 4}, \quad t>t_{d},
$$

where $\gamma<1$.

When annealing for long times (and/or high temperatures) the surface-trap density $N_{\mathbf{s t}}$ may also decrease through other mechanisms, especially by the introduction of diffusing species $X$ or other species from the outside (either from the metal or the ambient).

The stress and anneal data in Fig. 5 was further evaluated and $R_{1}^{\prime}=0.85$ was found during degradation. The surface-trap density $N_{r}$ during annealing is plotted against $\left[t^{1 / 4}-\left(t-t_{d}\right)^{1 / 4}\right]$ in Fig. 10。 It is shown that only a fraction $\gamma=0.32$ of the created surface traps was annealed out as suggested above.

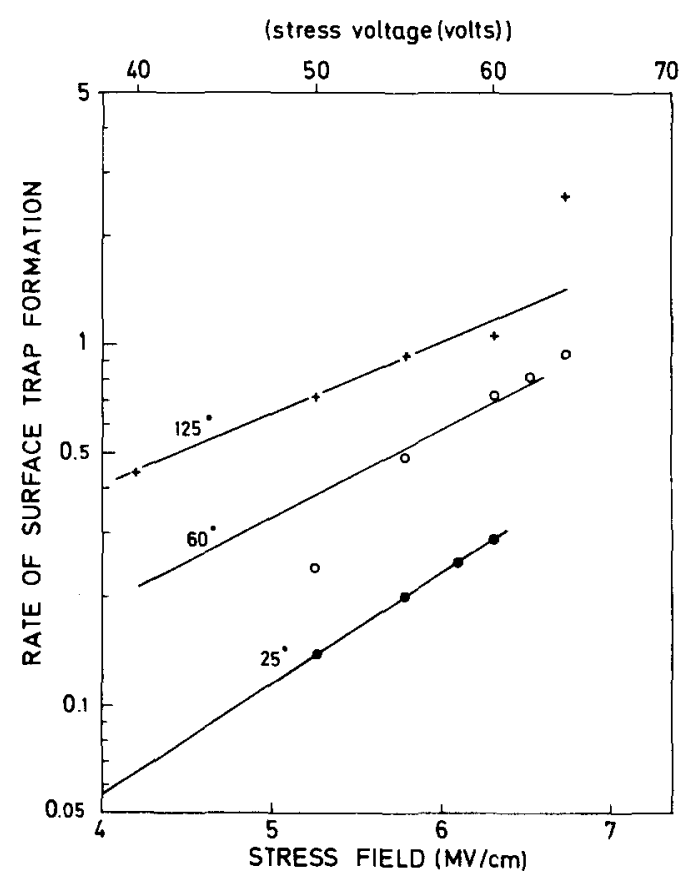

FIG. 9. The relative rate of surface-trap formation $R_{1}^{\prime}\left(t^{1 / 4} \mathrm{re}-\right.$ gion) is plotted against the NBS field for the three different NBS temperatures. 


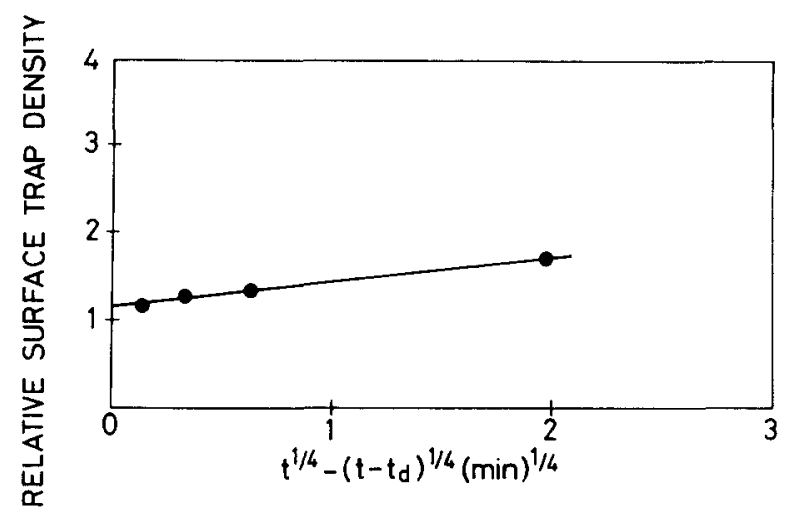

FIG. 10. The relative surface-trap density is plotted against $t^{1 / 4}-\left(t-t_{d}\right)^{1 / 4}$, where $t_{d}$ is the stress time $\left(t_{d}=15 \mathrm{~min}\right)$ and $t$ is the time elapsed since start of NBS.

\section{DEGRADATION MECHANISMS AT HIGH FIELDS}

For high stress voltages, particularly at low temperatures, $N_{r}$ appears to increase linearly with time. This is clearly shown in Fig. 3. A linear rate constant $R_{2}$ was defined for the surface-trap growth in this NBS region. The rate constant $R_{2}$ appears to be independent of stress temperature but strongly dependent of stress voltage which suggests that the rate is limited by a tunneling process. Such a process may be hole tunneling from the silicon valence band into silicon dioxide hole traps (see Fig. 11).

$$
\text { (oxide defect) }+h^{+} \rightleftharpoons \text { (oxide defect) }{ }^{*} \text {. }
$$

The charged oxide defect must then be able to create a surface trap. This is certainly possible, since it has been observed to happen during irradiation of MOS structures. Kjar and Nichols ${ }^{19}$ have clearly shown that surface traps are created with the same rate as oxide charge during irradiation of MOS structures. Powell and Derbenwick ${ }^{20}$ have further shown that such irradiation effects can occur as a result of hole injection only. It is therefore clear that hole trapping close to the Si$\mathrm{SiO}_{2}$ interface creates surface traps, although the mechanisms is not known.

In this model the trap formation rate would be proportional to the tunneling probability, that is,

$$
R_{2}=\frac{\partial N_{s t}}{\partial t} \sim \exp \left(-2 \chi x_{t}\right)=\exp \left(-E_{0} / E\right)
$$

where $\chi$ is the imaginary wave vector for electrons in the oxide and $x_{t}$ is the tunneling distance. In the actual part of the oxide forbidden band, $\chi$ is relatively independent of energy and equal to about ${ }^{21} 4.95 \times 10^{9} \mathrm{~m}^{-1}$.

According to $\mathrm{Eq}$. (10), we have plotted $\log R_{2}^{\prime}$ versus $1 / E$ with data taken at two temperatures in Fig. 12. From the slope of the line in Fig. 12 we obtain $E_{0}$ $=2 \times 10^{10} \mathrm{~V} / \mathrm{m}$.

From the observed field dependence of the trap formation rate we may thus estimate the trap depth. In Fig. 11 we find $X_{t}=\left(\phi_{t}-\phi_{1}\right) / E$, or from Eq. (10) $E_{0}$ $=2 \chi\left(\phi_{t}-\phi_{1}\right)$, which together with our experimental value of $E_{0}$ gives $\phi_{t}-\phi_{1}=2.0 \mathrm{eV}$. Using $\phi_{1}=4.2 \mathrm{eV}$, the

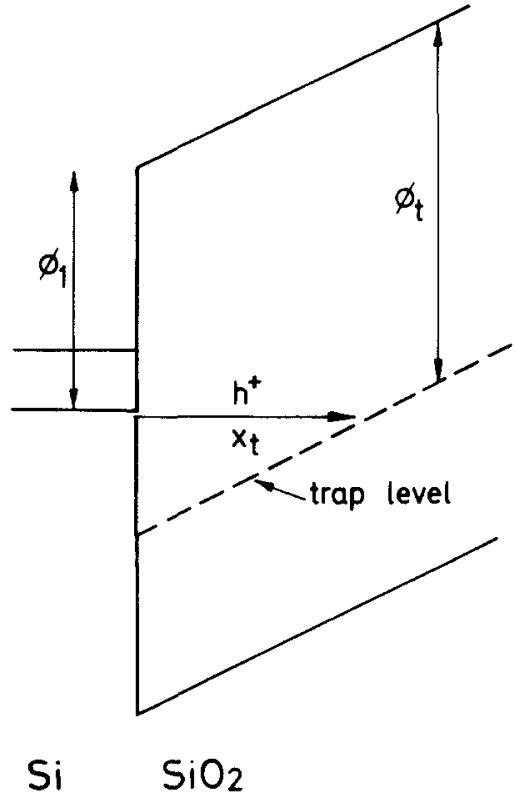

FIG. 11. Estimation of the observed hole trap level $\phi_{t}$ during NBS at high fields ( $t$ region), $\phi_{1}$ is the $\mathrm{Si}-\mathrm{SiO}_{2}$ barrier height. $X_{t}$ is the tunneling distance.

trap depth $\phi_{t}$ is estimated to be about $6.2 \mathrm{eV}$ below the oxide conduction band.

We thus suggest that holes are injected and trapped in the oxide and that these holes then create surface traps. How the holes create the traps in our case or in the ir radiation case is not known.

The lowest field at which this effect is observed $(600$ $\mathrm{MV} / \mathrm{m}$ ) is considerably higher than the fields used in the earlier studies. ${ }^{4,5}$ This effect has therefore not been observed by these workers. However, Nakagiri ${ }^{22}$ has reported a rapid increase of the surface-trap density after shortly-applied fields above $700 \mathrm{MV} / \mathrm{m}$. This increase shows a field dependence as strong as ours and

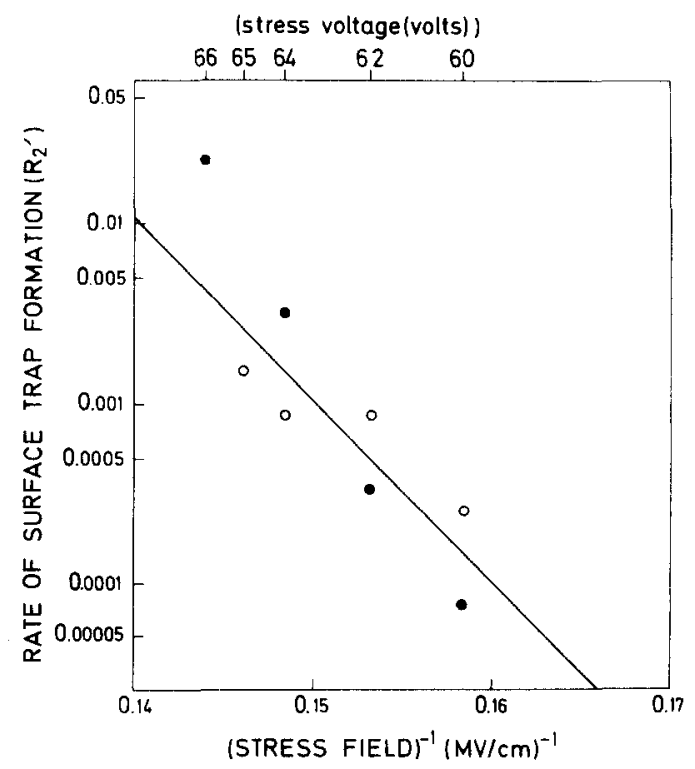

FIG. 12. The relative rate of surface-trap formation $R_{2}^{\prime}$ (linear region) is plotted against the inverse of the NBS field for two different NBS temperatures $\left(25\right.$ and $\left.60^{\circ} \mathrm{C}\right)$. 


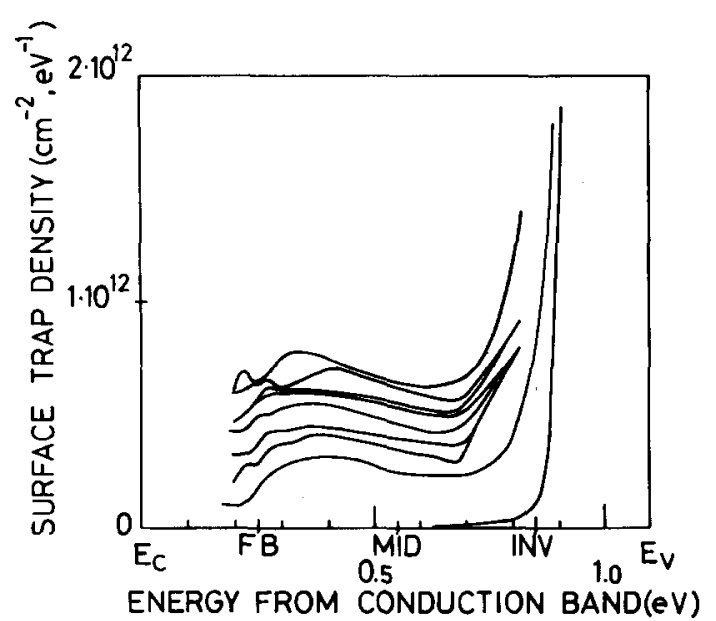

FIG. 13. A full evaluation of the increase of the surface-trap density after NBS at $125^{\circ} \mathrm{C},-55 \mathrm{~V}(5.8 \mathrm{MV} / \mathrm{cm})$. These NBS conditions correspond to the $t^{1 / 4}$ region (stress time: $0,3,7$, $12,20,35,50,80$, and $120 \mathrm{~min}$.

was also independent of temperature from 77 to $473 \mathrm{~K}$. Nakagiri observed this effect in both polarities, but somewhat higher fields were needed with positive bias. The observations of Nakagiri thus support our results.

\section{ENERGY DISTRIBUTION OF SURFACE-TRAP DENSITIES}

To shorten time-consuming data evaluation, only the midband surface-trap density has been studied so far. However, much more information may be obtained if the energy distribution of the surface-trap density is calculated. These calculations are particularly pertinent since we have observed two different types of surface-trap formation with different time dependences. Also, Goetzberger et al. ${ }^{5}$ observed that two independent peaks of surface-trap density emerged at different rates for different stress voltages.

The energy distribution of the surface-trap density is shown for two different NBS conditions in Figs. 13 and 14. In Fig. 13 the sample was stressed with $-55 \mathrm{~V}$ at $125^{\circ} \mathrm{C}$, i. e. , well inside the region with the $t^{1 / 4}$-related rate. The number of surface traps are evenly distributed across the energy range, except for a-moderate peak just below flatband. Also during stress, the number of surface traps increased evenly at all energy levels.

In Fig. 14 the sample was stressed at RT with -64 $\mathrm{V}$, i. e., the growth is linear with time. In this case uneven surface-trap distribution and growth was observed. The moderate flatband peak is still detected but it is masked by a fast-growing shoulder that "spreads" towards flatband from lower energies. It was not possible to determine whether the flatband peak possibly increased as $t^{1 / 4}$ because the fast-growing shoulder influenced the growth too much, even at flatband.

\section{MNOS DEGRADATION}

As stated in Sec. I, MNOS structures degrade upon high-field stress. This is of considerable importance since an MNOS memory device is normally operated at very high fields during the write and erase cycles. The most important form of degradation is a large thresholdvoltage shift caused by an increase of the surface-trap density. In order to investigate the degradation of MNOS structures we studied the rate of surface-trap formation in samples operated repeatedly at high alternating write/erase fields. This degradation was then compared with the NBS effect of MOS devices.

There are two differences between MOS and MNOS structures that are of importance when comparing them at high-field stress. First, changes in the "fixed charge" due to stress may not be observed in MNOS devices. This is because the flatband voltage is variable, the effect that makes the MNOS device useful as a memory. The flatband voltage is determined by the charge in the insulator and by the surface-charge density. The "fixed charge" cannot be separated from the "information charge" that is injected to the memory by the erase and write pulses. However, changes in the surface-trap density may be observed as before.

Second, the stored charge affects the electric field in the insulator, particularly in the oxide at the silicon surface. This field is determined by the gate voltage and the stored charge. ${ }^{23}$ Therefore, we are not interested in studying dc stress of MNOS structures, since the gate voltage injects charges into the insulator which considerably reduces the field at the silicon surface to an undetermined value. Instead we stress the MNOS device with an alternating write/erase square wave. Also, in this case the electric field is rather complicated, ${ }^{1}$ but with a proper pulse length the average field is approximately determined by the gate voltage.

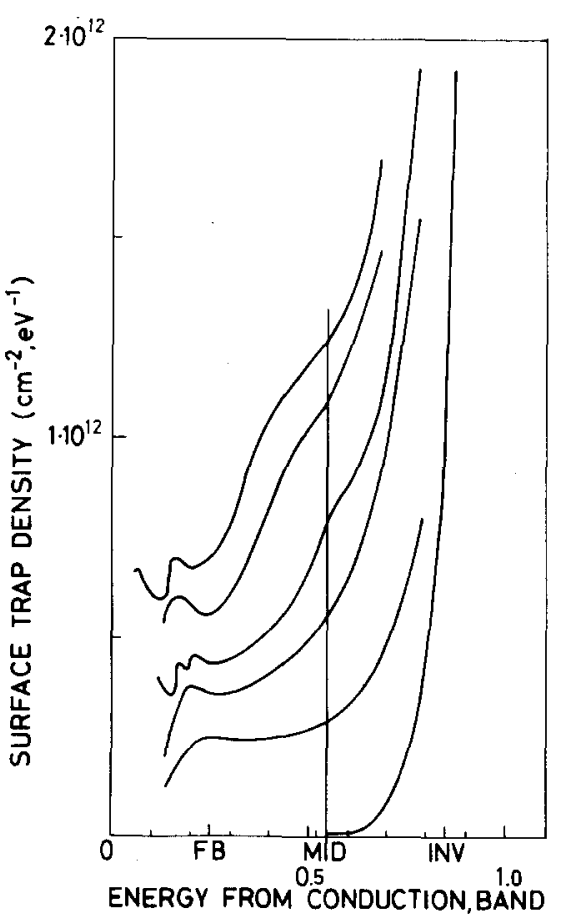

(eV)

FIG. 14. A full evaluation of the increase of the surface-trap density after NBS at $25^{\circ} \mathrm{C},-64 \mathrm{~V}(6.7 \mathrm{MV} / \mathrm{cm})$. NBS conditions correspond to the $t$ region (stress time: $0,5,10,15,25$, and $35 \mathrm{~min}$. ) 


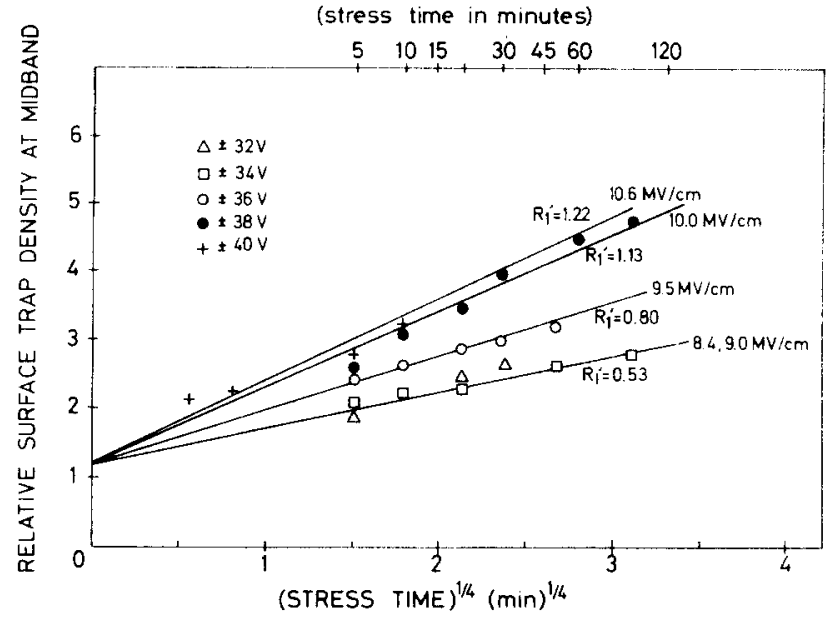

FIG. 15. Relative surface-trap density at midband plotted against (stress time $)^{1 / 4}$ for different write/erase pulse amplitudes at room temperature for MNOS sample CM-54.

These facts complicate the degradation measurements on MNOS samples. We see that even different write characteristics between samples will give them different degradation properties since the average oxide field will be different.

Let us discuss the measurement on one particular MNOS sample (CM-54). This sample is very similar to one of the samples (CM-55) in Ref. 6. The oxide thickness is $22 \AA$ and the nitride thickness is $640 \AA$. Typical write/erase cycles are $\pm 32 \mathrm{~V}, 10 \mathrm{~ms}$, giving a threshold window of $8 \mathrm{~V}\left(V_{T}= \pm 4 \mathrm{~V}\right)$. Degradation was investigated by stressing the device with a square wave with an amplitude of \pm 32 to $\pm 40 \mathrm{~V}$ and a period of $20 \mathrm{~ms}$ corresponding to repeated $10-\mathrm{ms}$ write/erase pulses. The temperature region $25-125^{\circ} \mathrm{C}$ was investigated. Before recording of the $\mathrm{C}-\mathrm{V}$ curves, a small voltage pulse moved the flatband voltage to $0 \mathrm{~V}$ in order to minimize the effect of nitride currents on the quasistatic $\mathrm{C}-\mathrm{V}$ measurement. As before, changes in the relative surface-trap density at midband were studied.

The observed time dependence of the surface-trap formation exhibits a $t^{1 / 4}$ time dependence but not a linear dependence, as shown in Fig. 15. This indicates the same mechanism that causes the $t^{1 / 4}$ behavior of the MOS devices, and a further comparison may be of interest. From Fig. 15 we get a rate constant $R_{1}^{\prime}=1.13$ at a stress voltage of $38 \mathrm{~V}$. This corresponds to an oxide field of about $1000 \mathrm{MV} / \mathrm{m}$ [taking $E_{\mathrm{ox}} \approx\left(\epsilon_{N} / \epsilon_{\mathrm{ox}}\right)$ $\times\left(V_{s} / t_{N}\right)$ ]. To compare this result with the MOS results we extrapolate the curves in Fig。 9 to $1000 \mathrm{MV} / \mathrm{m}$ and obtain $R_{1}^{\prime} \approx 3$ for the MOS device. These two values are of the same order of magnitude $\left(R_{1} \approx 6.5 \times 10^{15}\right.$ $\left.\mathrm{m}^{-2} \mathrm{eV}^{-1} \min ^{-1 / 4}\right)$.

By plotting $\log R_{1}^{\prime}$ against the stress voltage we may determine the constant $a$ in $\mathrm{Eq}$. (6). Using the data in Fig. 15 we get $a=2.7 \AA$, which again is about equal the value found in MOS structures.

We thus conclude that the surface trap formation in MNOS structures is caused by the same reaction that causes the observed $t^{1 / 4}$ part of the NBS effect in MOS structures. We therefore expect the diffusion-controlled model proposed above to be valid also for MNOS degradation. (The model must of course be modified since part of the diffusion takes place in the silicon nitride.)

One important conclusion is then that we may use known experimental data from NBS measurements on MOS devices in order to find methods of minimizing the degradation effects in MNOS devices

We mentioned above that measurements were performed at different temperatures. However, we were not able to evaluate the temperature dependence of $R_{1}$ because of lack of reproducibility of these measurements.

With an oxide field of $1000 \mathrm{MV} / \mathrm{m}$ one might expect the degradation of MNOS devices at room temperature to have a linear time dependence. The fact that we do not observe a linear time dependence is, however, in agreement with the proposed tunneling model. In a MNOS device the oxide is very thin and tunneling of holes from silicon into traps located in the oxide requires a certain oxide thickness as shown in Fig. 11. Tunneling to traps located in the nitride are not supposed to create surface traps. For an oxide field of $1000 \mathrm{MV} / \mathrm{m}$, a trap depth of $6 \mathrm{eV}$ would yield a tunneling distance of $20 \AA$ which is about equal to our oxide thickness. Therefore most holes will tunnel to nitride traps and hence not create any surface traps. However, in MNOS structures with oxide thickness much larger than $20 \AA$ we would expect the tunneling effect to be important, and such devices are thus expected to degrade faster than devices with thinner oxide layers.

It may be of interest to estimate the number of allowed write/erase cycles on our MNOS devices for comparison with other results. ${ }^{1-3}$ Assume that we allow the increase of the surface-trap density to cause a $1-V$ threshold-voltage shift. This corresponds to an increase of the surface-trap density of about $\Delta N_{\mathrm{st}}=1.7$ $\times 10^{12} \mathrm{~cm}^{-2} \mathrm{eV}^{-1}$ or $\Delta N_{r}=3$. At a $32-V$ stress voltage we get $\Delta N_{r}=3$ after $55000 \mathrm{sec}$ (Fig. 15) which corresponds to $3 \times 10^{6}$ write/erase cycles. It should also be noted that we expect this number of pulses to be lower at higher temperatures.

\section{CONCLUSIONS}

The process of surface-trap formation at the $\mathrm{Si}-\mathrm{SiO}_{2}$ interface during negative-bias stress has been studied. This is one of the most important degradation processes in MNOS memory devices during repeated write/erase cycling. This process has been characterized through studies of the surface-trap formation rate at different stress fields and temperatures. However, because of the difficulties in accurately determining the oxide field in MNOS devices we have chosen to evaluate this process through negative-bias-stress measurements on MOS devices.

Two mechanisms were observed to create new surface traps during NBS. The first mechanism appears at moderate fields and is believed to be diffusion controlled. The time dependence of such a process was shown to agree with experiments. The other mechanism is dominant at high fields $(>6.3 \mathrm{MV} / \mathrm{cm})$ where the 
formation of surface traps was shown to increase very rapidly with the applied field independent of stress temperature. This second mechanism is believed to be controlled by tunneling injection and trapping of holes but requires further clarification regarding the atomic model.

Finally, the NBS behavior of MOS capacitors was compared with the degradation behavior of MNOS capacitors and it was shown that the MNOS devices followed the diffusion-controlled mechanism discussed above.

\section{APPENDIX}

Consider a surface at $z=0$, at which a chemical reaction takes place. This reaction is schematically shown in Eq. (3). When the surface defect is electrically activated, a diffusing species leaves the defect site at which a surface trap and a surface charge are left in fixed positions close to each other.

The rate of surface-trap formation may then be written

$$
\frac{\partial N_{\mathrm{st}}}{\partial t}=A\left(N_{D}-N_{\mathrm{st}}\right)-B N_{\mathrm{st}} C_{X i},
$$

where $N_{D}$ is the initial concentration of surface defects, $C_{X i}$ is the concentration of the species $X$ at the interface, and $A$ and $B$ are field-dependent rate constants. If this process is diffusion limited rather than reaction-rate limited, the surface reaction will be in quasiequilibrium, giving

$$
C_{X i} N_{\mathrm{st}} \approx(A / B) N_{D}
$$

Here $N_{D}$ may be considered constant if we assume that the reaction is always far from saturation $\left(N_{s t} \ll N_{D}\right)$.

Diffusion control means that the rate of $N_{\text {st }}$ growth is controlled by the diffusion of $X$ away from the surface

$$
\frac{\partial N_{\mathrm{st}}}{\partial t}=\Psi_{X}
$$

$\Psi_{X}$ is the flow by diffusion of the species $X$ from the interface into the oxide

$$
\Psi_{X}=-D \frac{\partial C_{X}}{\partial z},
$$

where $D$ is the diffusion constant of $X$ and $\partial C_{X} / \partial z$ is the concentration gradient of $X$ in the oxide at a distance $z$ from the interface.

The diffusion rate is given by the diffusion equation

$$
\frac{\partial C_{X}}{\partial t}=D \frac{\partial^{2} C_{X}}{\partial z^{2}}
$$

where $C_{X}(z, t)$ is the concentration of $X\left[C_{X i}=C(0, t)\right]$. This equation may be solved by Laplace transforms, giving the Laplace transform of the concentration of $X$,

$$
\tilde{C}_{X}(z, s)=\tilde{C}_{1} \exp \left[-(s / D)^{1 / 2} z\right]+\tilde{C}_{2} \exp \left[(s / D)^{1 / 2} z\right],
$$

with $C_{X}(z, t)=0$ for $t<0$. Assuming $C_{X}(\infty, t)=0$, we get $\widetilde{C}_{2}=0$ and $\widetilde{C}_{1}=\tilde{C}_{X}(0, s)=\tilde{C}_{X i}(s)$. Note that this assumption is valid only if the oxide may be considered infinitely thick, i.e., if $W^{2} / 4 D t>1$ where $W$ is the oxide thickness.
The Laplace transform of the flow of $X$ from the surface is obtained from Eqs. (A4) and (A6) as

$$
\tilde{\Psi}_{X}=(D s)^{1 / 2} \tilde{C}_{X} .
$$

One solution to this system of equations is

$$
N_{\mathrm{st}}=R_{1} t^{1 / 4} \text {. }
$$

To see that this is true, we use the Laplace transform of $N_{s t}$, which is ${ }^{24} R_{1} \Gamma(5 / 4) s^{-5 / 4}$, together with Eqs. (A3) and (A7) and get

$$
\widetilde{C}_{X i}=R_{1} \Gamma(5 / 4) D^{-1 / 2} S^{-3 / 4} \text {. }
$$

The inverse Laplace transform of this gives

$$
C_{X i}=\frac{R_{1} \Gamma(5 / 4)}{(D)^{1 / 2} \Gamma(3 / 4)} t^{-1 / 4}
$$

By finally inserting $C_{X i}$ from Eq. (A10) and $N_{s t}$ from Eq. (A8) into Eq. (A2) we find that the above solution in $\mathrm{Eq}$. (A8) is true with

$$
R_{1}=\left(\frac{A N_{D}(D)^{1 / 2}}{B}\right)^{1 / 2}\left(\frac{\Gamma(3 / 4)}{\Gamma(5 / 4)}\right)^{1 / 2} \approx 1.16\left(\frac{A N_{D}(D)^{1 / 2}}{B}\right)^{1 / 2}
$$

$A / B$ is the equilibrium constant for the process (3) and can be written

$$
\frac{A}{B}=\left(\frac{A}{B}\right)_{0} \exp \left(-\frac{\Delta H}{k T}\right),
$$

where $\Delta H$ is the enthalpy of process (3). Assuming that the surface charge is located in the oxide at a distance $a$ from the silicon surface, we will expect the enthalpy of the process to decrease with $q a E_{\text {ox }}$ in an electric field $E_{\text {ox }}$. The quantity $q a E_{\text {ox }}$ is the energy gained by an electron moving from the oxide charge position into silicon. $\Delta H$ may thus be written

$$
\Delta H=q\left(\phi_{A B}-a E_{\text {ox }}\right),
$$

where $q \phi_{A B}$ is the zero-field value of $\Delta H$.

The diffusion coefficient $D$ is expected to have the following form:

$$
D=D_{0} \exp \left(-q \phi_{D} / k T\right),
$$

where $q \phi_{D}$ is the activation energy for $D$. The rate constant $R_{1}$ now becomes

$$
\begin{aligned}
R_{1}= & 1.16\left[(A / B){ }_{0} N_{D}\right]^{1 / 2} D_{0}^{1 / 4} \\
& \times \exp \left\{-(q / k T)\left[\left(\frac{1}{2} \phi_{A B}+\frac{1}{4} \phi_{D}\right)-\frac{1}{2} a E_{\mathrm{ox}}\right]\right\} .
\end{aligned}
$$

This equation predicts the field and temperature dependence of $R_{1}$ and agrees with Eq. (6) with

$$
\phi_{0}=\frac{1}{2} \phi_{A B}+\frac{1}{4} \phi_{D} \text {. }
$$

Earlier we determined $a=3.2 \AA$ and $\phi_{0}=0.3 \mathrm{eV}, \phi_{D}$ is expected to be about $0.3 \mathrm{eV}{ }^{18}$ so $\phi_{0}=0.3 \mathrm{eV}$ is not unreasonable, making $\phi_{A B}>0$ in Eq. (A16).

If the production of $X$ at the surface is stopped (by making $A=0$ ) after a certain time $t_{d}$ the species $X$ diffuses back to the surface and recombines with the defect site. This process is called annealing. If this annealing takes place at the same temperature as the 
degradation, we may calculate its time dependence as a continuation of the above calculation [Eq. (A8)].

Thus, for $0<1<l_{d}, C_{X i}$ is given by Eq. (A9), and, for $/ t_{d}, C_{X i} \approx 0$ according to Eq. (A2) with $A=0, \widetilde{C}_{X i}$ may therefore be written ${ }^{24}$ as

$$
\tilde{C}_{X i}=R_{1} \Gamma(5 / 4) D^{-1 / 2} s^{-3 / 4}\left[1-\exp \left(-s t_{d}\right)\right] \text {. }
$$

As above, we find, by using Eqs. (A3) and (A7), that

$$
N_{\mathrm{st}}=R_{1}\left[t^{1 / 4}-\left(t-t_{d}\right)^{1 / 4}\right], \quad t>t_{d}
$$

IJ.R. Cricchi and W.D. Reed, Jr., Proc. 9th Annual Reliability Physics Symposium, 1971, p. 1 (unpublished).

${ }^{2}$ M.H. Woods and J.W. Tuska, Proc. 10th Annual Reliability Physics Symposium, 1972, p. 120 (unpublished).

${ }^{3}$ J.R. Cricchi, F.C. Blaha, M.D. Fitzpatrick, and F.M. Sciulli, IEDM 1975 Tech. Dig. 459.

${ }^{4}$ B. E. Deal, M. Sklar, A.S. Grove, and E.H.Snow, J. Electrochem. Soc. 114, 266 (1967).

${ }^{5}$ A. Goetzberger, A.D. Lopez, and R.J. Strain, J. Electrochem. Soc. 120, 90 (1973).

${ }^{6}$ L. Lundkvist, C. Svensson, and B. Hansson, Solid-State Electron. 19, 221 (1976).

${ }^{7}$ M. Kuhn, Solid-State Electron. 13, 873 (1970)。

${ }^{8}$ A.D. Lopez, Rev. Sci. Instrum. 44, 200 (1972).
${ }^{9}$ C.N. Berglund, IEEE Trans. Electron. Devices ED-13, 701 (1966).

${ }^{10}$ R. H. Walden, J. Appl. Phys. 43, 1178 (1972).

${ }^{11}$ E. F. Nicollian, C.N. Berglund, P.F. Schmidt, and J。M. Andrews, J. Appl. Phys. 42, 5654 (1971).

${ }^{12}$ K. H. Beckman and N.J. Harrick, J. Electrochem. Soc. 118,614 (1971).

${ }^{13} \mathrm{G}$. L. Holmberg, A.B. Kuper, and F.D. Miraldi, J。 Elcetrochem. Soc. 117, 677 (1970).

${ }^{14} \Lambda_{\text {。 }} \mathrm{G}$. Revesz, IEEE Trans. Nucl. Sci. NS-18 (No. 12), 11:3 (1971).

${ }^{15} \mathrm{E}$. Kooi, Philips Res. Rep. 20, 528 (1965).

${ }^{16} \mathrm{~F}$. Montillo and P. Balk, J. Electrochem. Soc. 118, 146:3 (1971).

${ }^{17}$ J.P. Nitchell and D.G. Denure, Solid-State Electron 16 , 825 (1973).

${ }^{18}$ R.W. Lee, R.C. Frank, and D.E. Swets, J. Chem. Phys. 36, $1062(1962)$.

${ }^{19}$ R. A. Kjar and D. K. Nichols, IEEE Trans. Nucl. Sci。 NS-22, 2193 (1975).

${ }^{20}$ R. J. Powell and G. F. Derbenwick, IEEE Trans. Nucl. Sci. NS-18 (No. 12), 99 (1971).

${ }^{21} \mathrm{~J}$. Maserjian and G. Petersson, Appl. Phys. Lett. 25, 50 (1974).

${ }^{22}$ M. Nakagiri, Jpn. J. Appl. Phys. 13, 1610 (1974).

${ }^{23}$ K.I. Lundström and C. M. Svensson, IEEE Trans. Electron Devices ED-19, 826 (1972).

${ }^{24} \mathrm{M}$. Abramowitz and I. A. Stegun, Handbook of Mathematical Functions (Dover, New York, 1965), Chap. 29. 